\title{
A Two-Dimensional Spectrometer for Solar Observation and its recent modifications
}

\author{
Cornelia Bendlin
}

Institut für Astronomie und Astrophysik, Am Hubland, D-97074 Würzburg, Germany

\begin{abstract}
The instrument presented here is based on the combined use of a Universal Birefringent Filter (UBF) and a Fabry-Perot Interferometer (FPI) to obtain narrow-band filtergrams with a CCD. Scanning through a Fraunhofer line with a sufficient number of wavelength settings is accomplished within a few seconds by tuning only the FPI. The two-dimensional spectrometer in the German Vacuum Tower Telescope (VTT)/Tenerife was used to take observations of high spatial, spectral, and temporal resolution, yielding a wide range of results. Since recently, additional information on the solar magnetic field is obtained with it.
\end{abstract}

\section{The two-dimensional spectrometer}

A detailed description of the spectrometer in the VTT at the Observatorio del Teide on Tenerife is given in Bendlin et al. (1992), therefore a short explanation of the instrumental set-up will suffice here.

On the main light path from the prime focus of the telescope to the detector "CCD 2", the spectral bandwidth of the incoming light is more and more reduced by a series of filters. An interference filter with a full width at half maximum (FWHM) of its transmission curve of about $10 \AA$ serves as a prefilter for the UBF (FWHM $\approx 0.5 \AA$ ). Finally, the FPI acts as a narrow-band filter (FWHM $\approx$ $22 \mathrm{~m} \AA$ at $6303 \AA$ ). As it only has a very limited dispersion region ("free spectral range" $\approx 1 \AA$ ), the incoming light must be pre-dispersed to avoid overlapping of orders.

The narrow-band filtergrams are taken with CCD 2 which is coupled with an image intensifier to achieve short integration times.

A beam splitter cube behind the interference filter reflects part of the light onto another CCD ("CCD 1") which serves as an integral- or "white-"light camera. The achromats in these light paths were chosen to yield a spatial resolution of $0.2 \mathrm{arcsec} / \mathrm{pixel}$ on both detector chips. CCD 1 and CCD 2 accept the same field of view, and they are exposed strictly simultaneously, therefore the procedure used to remove the effects of image motion and distortions in the white-light images can also be applied to the narrow-band filtergrams.

Additional light paths with a continuum source, a laser source, and a photomultiplier, respectively, are important for the data reduction and for proper adjustment of the spectrometer. They may be used via movable mirrors, when needed. The two-dimensional spectrometer can be tuned to virtually any wave- 
length in the visible spectrum, and it is suitable for high spatial, spectral, and temporal resolution.

\section{Narrow-band filtergrams with an FPI}

An FPI essentially consists of two partially transmitting flat mirrors of high reflectivity which are parallel to each other. At each surface in the mirror gap, an incident beam of light is partially reflected and partially transmitted. If all the transmitted light beams are collected in one point, due to multiple-beam interference a passband is produced which is described by the Airy formula giving the ratio of transmitted to incident intensity:

$$
\frac{I^{(t)}}{I^{(i)}}=\frac{1}{1+F \cdot \sin ^{2} \frac{\delta}{2}}
$$

where

$F=$ const., $\delta=$ phase difference between successive beams $=(4 \pi n d \cos \theta) / \lambda$ with maximum values for $\delta=2 \pi \mathrm{m}$, the order of interference $\mathrm{m}$ being an integer, $\mathrm{n}=$ refractive index of the air in the mirror gap, $\mathrm{d}=$ mirror separation, $\theta=$ angle of incidence (in the mirror gap), $\lambda=$ wavelength.

For illustrations of the Airy function as well as for the theory of FPIs see e. g. Born \& Wolf (1959), Hernandez (1988), and Vaughan (1989).

The distance between neighbouring maxima of the Airy function is called "free spectral range" (FSR). The smaller it is the higher is the spectral resolution. Here, for observing the FeI line at 6302.5 $\AA$ an FSR of $1 \AA$ was chosen which yielded a smallest resolvable wavelength difference of approximately $22 \mathrm{~m} \AA$. Thus, a spectral resolution of about 300000 results.

The transmission curve of the UBF resembles a sinc ${ }^{2}$ function. During the observations, its main lobe remains centred upon the chosen line, while scanning through the line is performed with the FPI. With the proper mirror separation, one maximum of the FPI's passband corresponds to a wavelength position in the continuum near the Fraunhofer line. At this position, a narrow-band filtergram is taken. Then, the mirror separation is altered very quickly with piezoelectric elements. As a result, the maximum of the FPI's passband is shifted to another wavelength. At this position, the next narrow-band image is taken, and so on until the whole wavelength range of interest is scanned. This method of tuning is very fast. With an appropriate number of wavelength settings (and a suitable field of view), scanning through a line takes only a few seconds.

It is a long way, though, to obtain line profiles over a two-dimensional field of view from the narrow-band filtergrams. The data reduction is quite complex (Bendlin 1993), therefore only the main idea behind all the operations to be carried out can be given here: For each pixel, the spectrometer's passband contained in the narrow-band filtergrams taken with solar light has to be divided by its corresponding passband taken with the continuum source in the same wavelength range. Thus, a line profile can be reconstructed from the resulting (relative) intensities for each pixel in the two-dimensional field of view. 


\section{Data acquisition and observations}

In 1991, solar granulation near disk centre was observed in the FeI line at 6302.5 A. A time series of narrow-band images and white-light pictures was obtained simultaneously.

Here, a "scan" is defined as a set of 30 images. The narrow-band filtergrams were taken at (30) different wavelength settings with a wavelength difference $\delta \lambda \approx 18 \mathrm{~mA}$ between neighbouring images, thus a wavelength range of about $0.5 \AA$ was scanned around the chosen line.

With an image acquisition rate of about 11 to 12 images per second, less than 3 seconds were required for taking the whole scan. The time series obtained with the two-dimensional spectrometer consists of 128 repetitive scans with a time interval of $20 \mathrm{~s}$ between the start of one scan and that of the next. An exposure time of $40 \mathrm{~ms}$ proved to be sufficient.

The angular size of the field of view was about $26 \times 18 \operatorname{arcsec}^{2}$ with 0.2 arcsec per pixel on the CCD chip.

It is important to note that most of the observing parameters mentioned above can be varied over a wide range, therefore many ambitious observing programs may be tackled using this spectrometer.

\section{Results}

Out of the large number of results obtained so far (Bendlin 1993, Bendlin \& Volkmer 1993), only rather general examples will be presented here to illustrate the possibilities offered by the new instrument (see also Figure 1).

- As from the continuum to the line centre higher and higher atmospheric layers are observed, narrow-band filtergrams were used to follow individual structures in the vertical direction of the solar atmosphere.

- Velocity fields were derived from the Doppler shifts of the line profiles over the two-dimensional field of view. Since velocity fields were obtained at regular intervals of $20 \mathrm{~s}$ for all the scans of the time series, it was possible to remove contributions from the so-called 5 -minute oscillations by filtering in the Fourier domain.

- "Intensity images" were computed which give the residual intensities at fixed line depths, irrespective of wavelength shifts.

- The FWHM was computed for every profile. The variation of this line parameter over the two-dimensional field of view was also represented by images (which is feasible for other line parameters, too).

- The best white-light images out of each scan of the time series served for a movie and thus for monitoring the temporal development of individual structures in the solar atmosphere. As the time interval between taking successive images is known, horizontal velocities could also be obtained. 


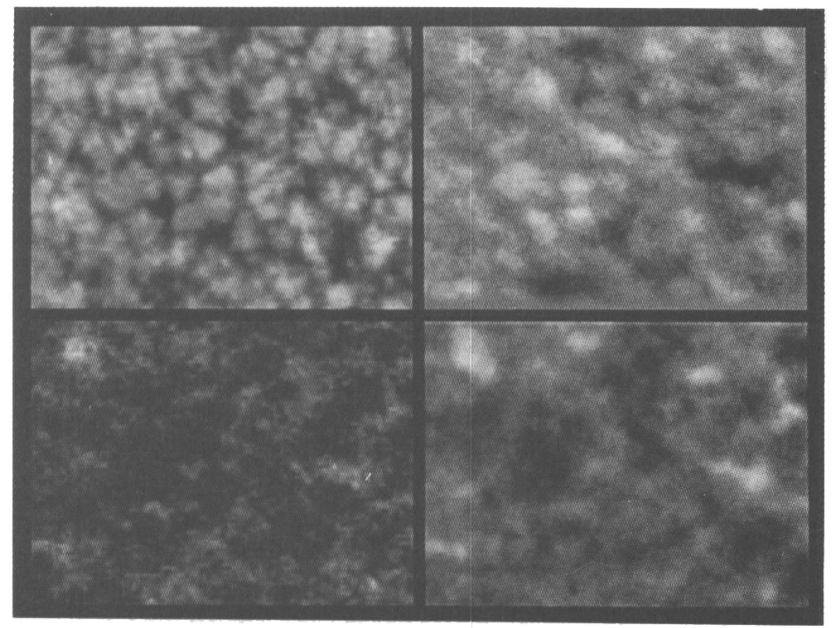

Figure 1. Upper left image: best image of a white-light scan; upper right: corresponding velocity field (corrected for 5-minute oscillations; scaling: medium shade of grey represents mean velocity $\bar{v}=0$, darker shades: downflows, lighter ones: upflows); lower left: FWHM of the corresponding line profiles (the darker the shades of grey the narrower the profiles); lower right: residual intensity of every line minimum, irrespective of wavelength shifts. Field of view: $21.8 \times 15.8 \operatorname{arcsec}^{2}$. 


\section{Recent modifications}

In 1992, the original set-up was modified. The main new component is a polarimeter with which Stokes-I and Stokes-V profiles and thus the longitudinal component of the magnetic field can be obtained. A schematic representation and a description of the spectro-polarimeter are given in Volkmer (1994).

It is possible, though, to use the instrument as a spectrometer again. For this purpose, the polarimeter needs to be exchanged for a suitable block of glass.

Acknowledgments. Thanks are due to $F$. Kneer for helpful advice and to R. Volkmer for his most effective participation in this project. Financial support was provided by the Deutsche Forschungsgemeinschaft through grants Kn 152/4-1 and Kn 152/4-2.

\section{Discussion}

J. P. Maillard: Is this instrument usable or planned to be used to do helioseismology?

C. Bendlin: Yes, it is. Results in this field may be expected in the near future.

\section{References}

Bendlin, C., Volkmer, R., Kneer, F. 1992, A\&A, 257, 817

Bendlin, C. 1993, Ph. D. Thesis, University of Göttingen, Germany

Bendlin, C., Volkmer, R. 1993, A\&A, 278, 601

Born, M., Wolf, E. 1959, Principles of Optics, Pergamon Press, London

Hernandez, G. 1988, Fabry-Perot Interferometers, Cambridge Studies in Modern Optics 3, Cambridge University Press, Cambridge

Vaughan, J. M. 1989, The Fabry-Perot Interferometer, Adam Hilger, Bristol Volkmer, R. 1994, Proceedings of the IAU-Coll. No. 149 